\title{
Comparative Analysis of Trapping Efficiencies between Gaussian and Laguerre-Gaussian beams for Optical Tweezers
}

\author{
Tasmia Farihba ${ }^{1}$, Ebad Zahir ${ }^{2}$ \\ ${ }^{1}$ (EEE Department, American International University - Bangladesh) \\ ${ }^{2}$ (Assistant Professor, EEE Department, American International University - Bangladesh)
}

\begin{abstract}
Using numerical calculations based on a mathematical model, this paper shows how the axial trapping efficiency $\left(Q_{z}\right)$ and the radial trapping efficiency $\left(Q_{r}\right)$ of optical tweezers depend on specific parameters such as size of the dielectric particle (r), numerical aperture of the microscope objective (NA), refractive index of the dielectric particle $\left(n_{p}\right)$ to be trapped and the refractive index of the surrounding medium $\left(n_{m}\right)$ in which the particle is being trapped. The model is used to calculate the trapping efficiencies and the forces acting on a particle. The calculations are done for two types of laser beams: Gaussian and LaguerreGausssian. Results for both types are then compared in terms of effective trapping. For all of the simulations, the particle being trapped is considered to be a micrometer-sized spherical polystyrene bead.
\end{abstract}

Keywords: Axial Trapping Efficiency, Gaussian Beams, Laguerre-Gaussian Beams, Optical Tweezers, Radial Trapping Efficiency, Sub-micron Dielectric Particles.

\section{Introduction}

Optical tweezers use light to trap, manipulate and position particles in three dimensions without any mechanical touch. By using the various effects of light, dielectric particles ranging in size from submicron to hundreds of microns can be successfully held by a beam (or beams) of light. Optical tweezers is a type of optical micro-manipulation and micro-fabrication system. Optical tweezers are scientific instruments that use a highlyfocused laser beam to trap, move and rotate microscopic individual dielectric particles in an aqueous medium. The forces exerted on the object result from the interaction between the laser and the refractive index mismatch of the object with its environment and are of the order of pico-newtons. [1]

\subsection{Principles of Optical Tweezers}

An optical trap is formed by tightly focusing a laser beam with an objective lens of high numerical aperture (NA). A dielectric particle near the focus will experience a force. Light can be described in terms of a stream of photons which are mass-less particles each carrying energy and momentum. When a light beam is incident on an interface, typically the surface of the trapped object, the beam is deflected due to reflection and/or refraction. The deflection of the beam induces changes to the momentum of the photons that the beam carries, and these momentum changes cause optical forces to be applied to the trapped object. It is noted that the momentum of the photons may be increased or decreased, depending on the beam intensity distribution; the optical forces may be either along or opposite to the direction of the incident photons. [2]

The optical forces can be classified into two types, the scattering force and the gradient force. The scattering force results from the reflection, scattering, and absorption of incident photons, and it is always along the direction of optical beam propagation. The gradient force arises as a result of a coherent interaction with light in which "the laser field polarizes the atom, and the polarized atom experiences a force in the gradient of an electromagnetic field". Gradient forces occur whenever a transparent material with a refractive index greater than its surrounding medium is placed within a light gradient. The gradient force acts in the direction of increasing light intensity, it pulls the object towards the position of the highest intensity and is proportional to the gradient of light intensity. [3] An optical trap or optical tweezers are essentially an optical field that can apply optical forces on the particles located in the field and, therefore, confine the positions of particles, as if the particles are "trapped" by the optical field. [2] Fig. 1 shows the origin of the two forces, gradient (lateral) force and scattering (axial) trapping force in optical tweezers. [4] A general experimental setup of optical tweezers with the most basic components is shown in Fig.2. [1] 


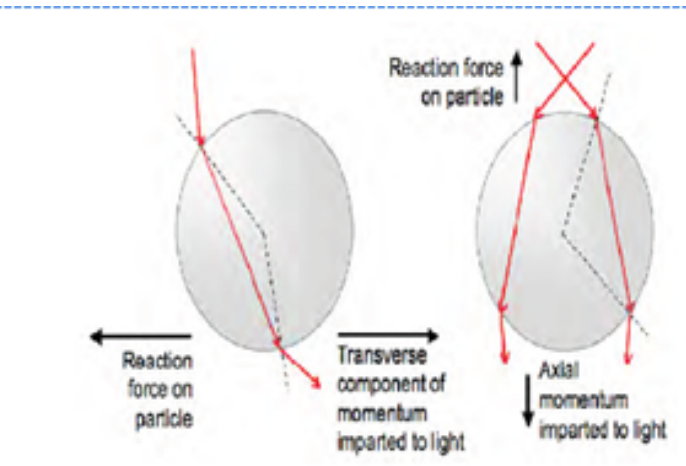

Fig. 1: Origin of axial and lateral trapping force in optical tweezers [4]

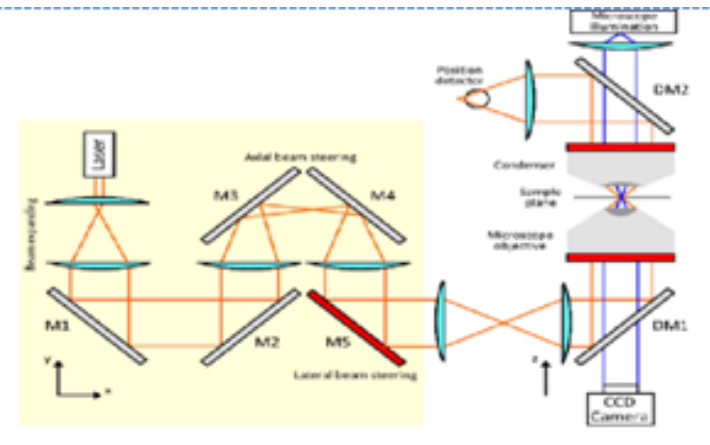

Fig. 2: A generic optical tweezer diagram with only the basic components [1]

A common way to quantify the contributions of these two forces in an optical trap is through a dimensionless factor known as trapping efficiency $(\mathrm{Q})$. To compare the optical forces obtained with respect to different optical beam powers, trapping efficiencies can be calculated which describes the efficiency of transferring the momentum from the light to the trapped object. [2] The efficiency of the trap can be described by a radial and an axial trapping efficiency, which are commonly measured by determining the maximum velocity at which the particle can be trapped through the surrounding fluid and still remain trapped by a given laser output power or by measuring the effective force constants. [5]

\section{Mathematical Model}

All calculations in this paper were done using a computational toolbox implemented in MATLAB for the computational modeling of optical tweezers. [6] The optical forces and torques that allow trapping and manipulation of microparticles in beams of light result from the transfer of momentum and angular momentum from the electromagnetic field to the particle- the particle alters the momentum or the angular momentum flux of the beam through scattering. [6] Therefore, if the scattering of the trapping beam by the trapped particle can be calculated, the optical force and torque can be calculated. [7] The toolbox can be useful for light scattering using either Lorenz-Mie theory or the T-matrix method. The toolbox describes the scattered fields using the Tmatrix method in which, as in generalized Lorenz-Mie theory (GLMT), the incident and scattered fields are expanded in terms of vector spherical wave functions (VSWFs). [6, 7] Unlike GLMT, the $T$-matrix method does not require the surface of the particle to be a constant surface in a separable coordinate system. The method is computationally well-suited for optical tweezers since the $T$-matrix for a given particle needs to be calculated only once. [7] The choice of coordinate system is spherical coordinates centered on the trapped particle. In their $T$-matrix calculations, the incoming and outgoing fields are expanded in terms of incoming and outgoing VSWFs:

$$
\begin{aligned}
& E_{\text {inc }}(r)=\sum_{n=1}^{\infty} \sum_{m=-n}^{n} a_{n m M_{n m}^{(2)}}(k r)+b_{n m} N_{n m}^{(2)}(k r) \\
& E_{\text {scat }}(r)=\sum_{n=1}^{\infty} \sum_{m=-n}^{n} p_{n m M_{n m}^{(1)}}(k r)+q_{n m} N_{n m}^{(1)}(k r)
\end{aligned}
$$

Where the VSWFs are: $M_{n m}^{(1,2)}(k r)=N_{n} h_{n}^{(1,2)} C_{n m}(\theta, \phi)$

$$
N_{n m}^{(1,2)}(k r)=\frac{h_{n}^{(1,2)}(k r)}{k r N_{n}} P_{n m}(\theta, \phi)+N_{n}\left(h_{n-1}^{1,2}(k r)-\frac{n h_{n}^{(1,2)}(k r)}{k r} B_{n m}\right)(\theta, \phi)
$$

Where, $h_{n}^{(1,2)}(k r)$ are spherical Hankel functions of the first and second kind, $N_{n}=[n(n+1)]^{-1 / 2}$ are normalization constants. $B_{n m}(\theta, \phi)=r \nabla Y_{n}^{m}(\theta, \phi), C_{n m}(\theta, \phi)=\nabla \times\left(r Y_{n}^{m}(\theta, \phi)\right)$ and $P_{n m}(\theta, \phi)=\hat{r} Y_{n}^{m}(\theta, \phi)$ are vector spherical harmonics. $Y_{n}^{m}(\theta, \phi)$ are normalized scalar spherical harmonics. The torque efficiency about the $z$-axis acting on the trapped particle is:

$$
\tau_{z}=\sum_{n=1}^{\infty} \sum_{m=-n}^{n} m\left(\left|a_{n m}\right|^{2}+\left|b_{n m}\right|^{2}-\left|p_{n m}\right|^{2}-\left|q_{n m}\right|^{2}\right) / P
$$

in units of $\hbar$ per photon, 


$$
P=\sum_{n=1}^{\infty} \sum_{m=-n}^{n}\left|a_{n m}\right|^{2}+\left|b_{n m}\right|^{2}
$$

is proportional to the incident power. The axial trapping efficiency, Q is:

$$
\begin{gathered}
Q=\frac{2}{P} \sum_{n=1}^{\infty} \sum_{m=-n}^{n} \frac{m}{n(n+1)} \operatorname{Re}\left(a_{n m}^{*} b_{n m}-p_{n m}^{*} q_{n m}\right)-\frac{1}{n+1}\left[\frac{n(n+2)(n-m+1)(n+m+1)}{(2 n+1)(2 n+3)}\right] \\
\left.a_{n m} a_{n+1, m}^{*}+b_{n m} b_{n+1, m}^{*}-p_{n m} p_{n+1, m}^{*}-q_{n m} q_{n+1, m}^{*}\right)
\end{gathered}
$$

The expansion coefficients $a_{n m}$ and $b_{n m}$ describing the incident beam are found by using an overdetermined point-matching theorem providing a stable and robust numerical performance and convergence. The expansion coefficients of the outgoing (i.e., scattered) field are found from the expansion coefficients of the incoming field using the $T$-matrix: $\mathrm{p}=\mathrm{Ta}$ where a and $\mathrm{p}$ are vectors formed from the expansion coefficients of the incident wave $\left(a_{n m}\right.$ and $\left.b_{n m}\right)$ and the scattered wave $\left(p_{n m}\right.$ and $\left.q_{n m}\right)$. $[6,7,8,9]$ It is sufficient to give the formulae for the $\mathrm{z}$-components of the fields. The same formulae can be used to calculate the $\mathrm{x}$ and $\mathrm{y}$ components of the optical force and torque, using 90 degree rotations of the coordinate system

\section{Results And Analysis}

Simulations of $\mathrm{Q}_{z}$ and $\mathrm{Q}_{\mathrm{r}}$ were carried out for different combinations of four parameters: size of the dielectric particle, numerical aperture of the microscope objective, refractive index of the dielectric particle to be trapped and the refractive index of the surrounding medium in which the particle is being trapped. The simulations were done for two types of laser beams: Gaussian and Laguerre-Gaussian. For all of the simulations, the particle being trapped was considered to be a micrometer-sized spherical polystyrene bead.The results will be compared for the two types of laser beams. Fig. 3 and Fig.4 shows how the trapping efficiencies $\mathrm{Q}_{\mathrm{z}}$ and $\mathrm{Q}_{\mathrm{r}}$ vary with displacement obtained using the toolbox. [6]

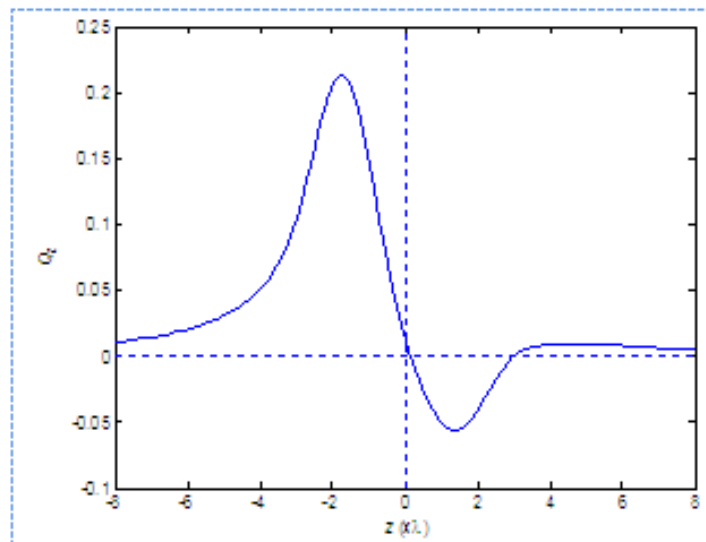

Fig. 3: The Axial trapping efficiency as a function of axial displacement

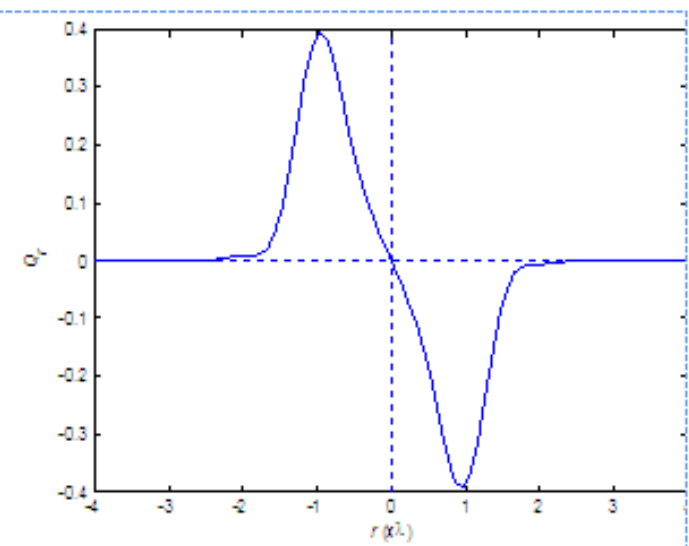

Fig. 4: The Axial trapping efficiency as a function of axial displacement

The following table below (Table-1) compares how the maximum axial trapping efficiency and the axial trapping efficiency vary when the refractive index of the medium is changed for both Gaussian beam and Laguerre-Gaussian beams:

Table-1

\begin{tabular}{|c|c|c|c|c|c|}
\hline \multirow{2}{*}{$\begin{array}{c}\text { Type of } \\
\text { Medium }\end{array}$} & $\begin{array}{c}\text { Refractive } \\
\text { Index }\end{array}$ & \multicolumn{2}{|c|}{ Gaussian beam } & \multicolumn{2}{c|}{$\begin{array}{c}\text { Laguerre-Gaussian } \\
\text { beam }\end{array}$} \\
\cline { 2 - 6 } & $\mathbf{n}_{\mathbf{m}}$ & $\mathbf{Q}_{\mathbf{z m a x}}$ & $\mathbf{Q}_{\mathbf{r m a x}}$ & $\mathbf{Q}_{\mathbf{z m a x}}$ & $\mathbf{Q}_{\mathbf{r m a x}}$ \\
\hline Water & 1.33 & 0.2138 & 0.3945 & 0.3384 & 0.2685 \\
\hline Silicon oil & 1.336 & 0.206 & 0.3854 & 0.3296 & 0.2654 \\
\hline $10 \%$ glucose & 1.3477 & 0.1911 & 0.3666 & 0.3129 & 0.2639 \\
\hline Ethanol & 1.361 & 0.1751 & 0.3438 & 0.2926 & 0.2591 \\
\hline 20\% glucose & 1.3635 & 0.1722 & 0.3394 & 0.2886 & 0.2576 \\
\hline $60 \%$ glucose & 1.4394 & 0.0947 & 0.2149 & 0.1596 & 0.0176 \\
\hline
\end{tabular}


In Table-1, $\mathrm{Q}_{z \max }$ and $\mathrm{Q}_{\mathrm{rmax}}$ are the maximum axial and radial trapping efficiencies respectively for each of the different mediums used for trapping the dielectric particle. In Fig. 5, the values of the maximum axial trapping efficiency are plotted against the refractive index of the medium. The blue curve shows $\mathrm{Q}_{\mathrm{zmax}}$ values for Gaussian beam and the red dotted lines shows the $\mathrm{Q}_{z \max }$ values for Laguerre-Gaussian beam. The graph shows the variation of $\mathrm{Q}_{\mathrm{zmax}}$ for both Gaussian and Laguerre-Gaussian when the refractive index of the surrounding medium $\left(\mathrm{n}_{\mathrm{m}}\right)$ is varied. It is observed that the value of the maximum efficiency for Laguerre-Gaussian beam is always higher than Gaussian beam. As the refractive index of the medium is increased, the axial efficiency starts to decrease for both Gaussian and Laguerre -Gaussian beams. A decrease in trapping efficiency suggests that the transfer of momentum from the source to the particle is poor and thus the particle cannot be trapped effectively.

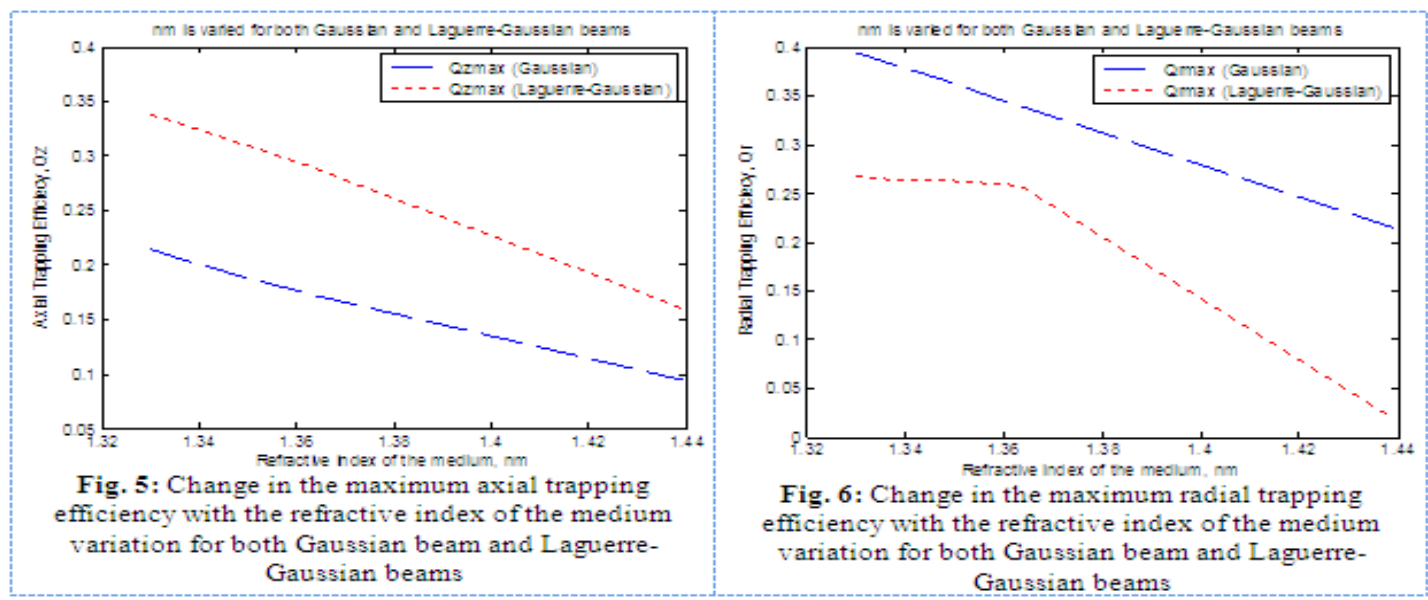

Fig.6 compares how the maximum radial trapping efficiency varies when the refractive index of the medium is varied for both Gaussian beam and Laguerre-Gaussian beams. The blue dotted lines show the $\mathrm{Q}_{\mathrm{rmax}}$ values for Gaussian beam and the red dotted lines show the $\mathrm{Q}_{\mathrm{rmax}}$ values for Laguerre-Gausssian beam. It can be seen from the figure that the maximum radial trapping efficiency obtained using Gaussian beam is higher than that from using Laguerre-Gaussian beam. As the refractive index of the medium increases, $\mathrm{Q}_{\mathrm{rmax}}$ starts to decrease. The slope of the curve shows that $\mathrm{Q}_{\mathrm{rmax}}$ decreases more drastically for Laguerre-Gaussian beam compared to Gaussian beam. The highest radial trapping efficiency is achieved when the medium is water $\left(\mathrm{n}_{\mathrm{m}}\right.$ $=1.33$ ).

The refractive index of the particle being trapped $\left(n_{p}\right)$ is then changed to observe how it affects the axial and the radial trapping efficiency for both Gaussian beam and Laguerre-Gaussian beams. The refractive index of the medium is kept constant at 1.33 which is water, NA is kept constant at 1.02; the maximum values of Qz and Qr are tabulated in Table-2. The values of $\mathrm{Q}_{z \max }$ are plotted against $n_{p}$ in Fig. 7 and $\mathrm{Q}_{\mathrm{rmax}}$ is plotted against $n_{p}$ in Fig. 8 for both Gaussian beam and Laguerre Gaussian beam. The blue dotted lines represent the $\mathrm{Q}_{z \max }$ values for Gaussian beam and the red dotted lines represent the $\mathrm{Q}_{z \max }$ values for Laguerre-Gaussian beam. Upon increasing the refractive index if the particle, the axial trapping efficiency for both Gaussian and Laguerre-Gaussian beam increase, as observed in Table 4 and in Fig. 7. The slope of the curve for LaguerreGaussian beam is steeper which means the axial trapping efficiency increases more sharply compared to Gaussian beam. However, when $n_{p}$ is increased beyond 1.6, Qz starts to decrease for Laguerre-Gaussian beam. In Fig. 4, radial trapping efficiency is seen to vary differently. As $n_{p}$ increases, $\mathrm{Q}_{\mathrm{rmax}}$ starts to increase for both Gaussian and Laguerre-Gaussian beam sharply but after $n_{p}$ is increased beyond 1.5, the increase in $\mathrm{Q}_{\mathrm{rmax}}$ for Laguerre-Gaussian is not as sharp as Gaussian. When $n_{p}$ is further increased above 1.65, $\mathrm{Q}_{\mathrm{rmax}}$ starts decreasing for both types of beam meaning the particle cannot be trapped in a stable trap anymore.

Table-2

\begin{tabular}{|c|c|c|c|c|}
\hline \multirow{2}{*}{$\begin{array}{c}\text { Refractive index of the } \\
\text { particle, }\left(\mathbf{n}_{\mathbf{p}}\right)\end{array}$} & \multicolumn{2}{|c|}{ Gaussian beam } & \multicolumn{2}{c|}{ Laguerre-Gaussian beam } \\
\cline { 2 - 5 } & $\mathbf{Q}_{\text {zmax }}$ & $\mathbf{Q}_{\mathbf{r m a x}}$ & $\mathbf{Q}_{\text {zmax }}$ & $\mathbf{Q}_{\text {rmax }}$ \\
\hline 1.39 & 0.03922 & 0.09976 & 0.06581 & 0.0831 \\
\hline 1.42 & 0.06196 & 0.1481 & 0.104 & 0.1246 \\
\hline 1.44 & 0.07801 & 0.179 & 0.1361 & 0.1511 \\
\hline 1.50 & 0.1295 & 0.2675 & 0.2198 & 0.2215 \\
\hline 1.59 & 0.2138 & 0.3945 & 0.3384 & 0.266 \\
\hline 1.62 & 0.2439 & 0.4217 & 0.3797 & 0.2844 \\
\hline 1.65 & 0.2749 & 0.4321 & 0.415 & 0.2931 \\
\hline 1.70 & 0.3275 & 0.3587 & 0.3703 & 0.2161 \\
\hline
\end{tabular}




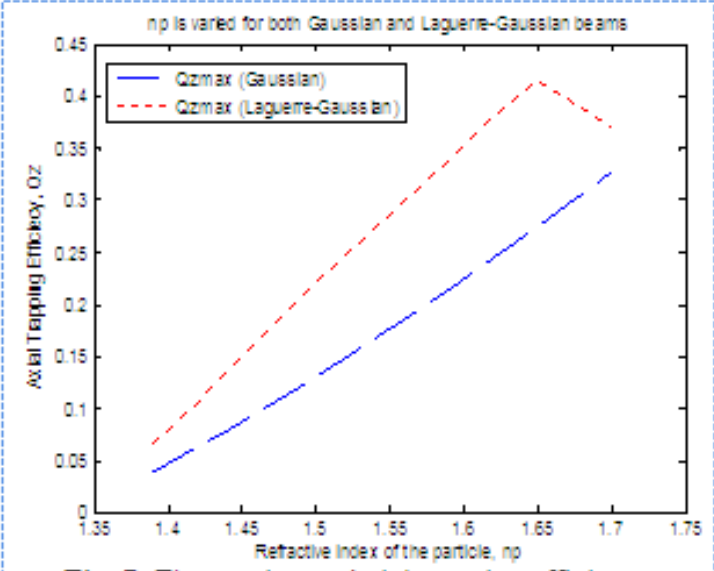

Fig. 7: The maximum Axial trapping efficiency $\left(Q_{\max }\right)$ when the refractive index of the particle is varied for both Gaussian beam and Laguerre-Gaussian beams.

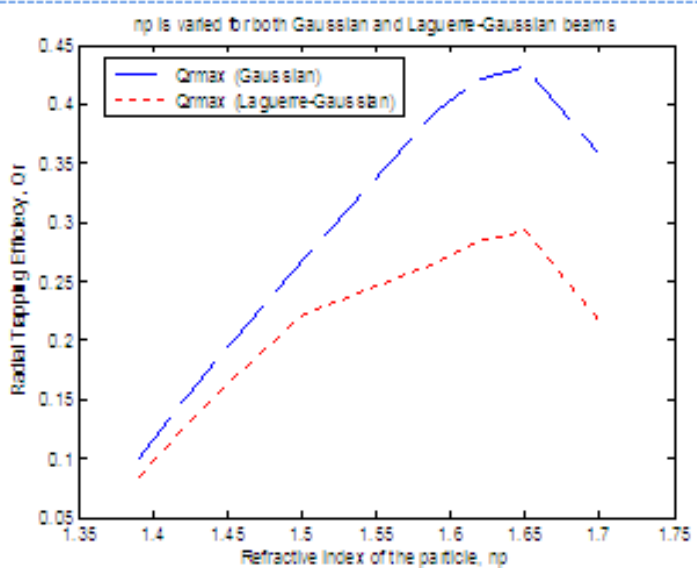

Fig. 8: The maximum Radial trapping efficiency

$\left(Q_{\max }\right)$ when the refractive index of the particle is varied for both Gaussian beam and Laguerre Gaussian beams.

Table-3 compares how the maximum axial trapping efficiency and radial trapping efficiency vary when the numerical aperture of the microscope objective is changed for Gaussian beam and Laguerre-Gaussian beams.

Table-3

\begin{tabular}{|c|c|c|c|c|}
\hline \multirow{2}{*}{$\begin{array}{c}\text { Numerical Aperture, } \\
\text { (NA) }\end{array}$} & \multicolumn{2}{|c|}{ Gaussian beam } & \multicolumn{2}{c|}{$\begin{array}{c}\text { Laguerre-Gaussian } \\
\text { beam }\end{array}$} \\
\cline { 2 - 5 } & $\mathbf{Q}_{\text {zmax }}$ & $\mathbf{Q}_{\text {rmax }}$ & $\mathbf{Q}_{\text {zmax }}$ & $\mathbf{Q}_{\text {rmax }}$ \\
\hline 1.02 & 0.2138 & 0.3945 & 0.3384 & 0.2685 \\
\hline 1.1 & 0.2283 & 0.4092 & 0.3535 & 0.2819 \\
\hline 1.15 & 0.2398 & 0.4164 & 0.3626 & 0.2834 \\
\hline 1.2 & 0.2524 & 0.421 & 0.3687 & 0.2844 \\
\hline 1.25 & 0.2643 & 0.4182 & 0.3637 & 0.2755 \\
\hline 1.3 & 0.2888 & 0.4204 & 0.3284 & 0.2387 \\
\hline 1.35 & 0.08996 & 0.11 & 0.09936 & 0.1033 \\
\hline 1.4 & 0.09009 & 0.11 & 0 & 0 \\
\hline 1.45 & 0.090009 & 0.1098 & 0 & 0 \\
\hline 1.5 & 0.090009 & 0.11 & 0 & 0 \\
\hline
\end{tabular}

From Table-3, values of $\mathrm{Q}_{\mathrm{zmax}}$ against NA and $\mathrm{Q}_{\mathrm{rmax}}$ against NA are plotted shown in Fig. 9 and Fig. 10 respectively. As NA of the microscope objective is increased from 1.02 to 1.3, both the trapping efficiencies Qz and Qr increase. When NA is further increased above 1.3, Qz and Qr both decrease drastically. For LaguerreGaussian, when NA is increased above 1.25, Qz and Qr both decrease. When NA is increased beyond 1.3, Q $\mathrm{Q}_{\mathrm{zmax}}$ and $\mathrm{Q}_{\mathrm{rmax}}$ both decrease drastically. Above $\mathrm{NA}=1.35$, the trapping efficiencies for Laguerre-Gaussian drops to zero.

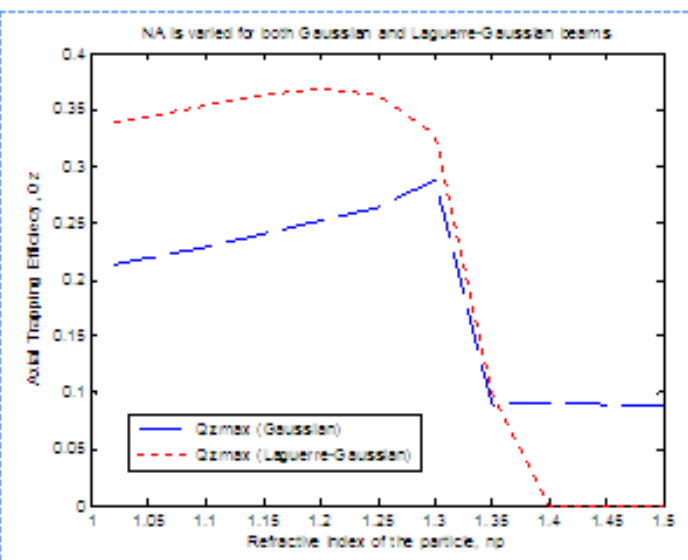

Fig. 9: The maximum Axial trapping efficiency $\left(Q_{\max }\right)$ when the numerical aperture of the microscope objective is varied for both Gaussian beam and Laguerre Gaussian beams.

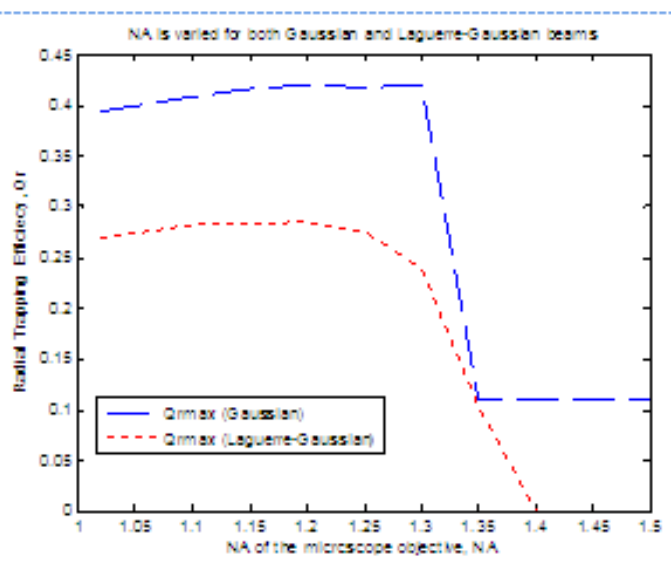

Fig. 10: The maximum Axial trapping efficiency

$\left(Q_{\max }\right)$ when the numerical aperture of the microscope objective is varied for both Gaussian beam and Laguerre Gaussian beams. 
Table-4 shows how the maximum axial trapping efficiency and radial trapping efficiency vary when the size (radius) of the particle is changed for both Gaussian beam and Laguerre-Gaussian beams. The values of $\mathrm{Q}_{z \max }$ are plotted against $n_{p}$ in Fig. 9 and $\mathrm{Q}_{\mathrm{rmax}}$ is plotted against $n_{p}$ in Fig. 10 for both Gaussian beam and Laguerre-Gaussian beam. The blue dotted lines represent the $\mathrm{Q}_{z \max }$ values for Gaussian beam and the red dotted lines represent the $\mathrm{Q}_{z \max }$ values for Laguerre-Gaussian beam. For very small particles, less then $1.5 \mu \mathrm{m}$, the trapping efficiencies are too poor suggesting that small particles cannot be trapped in a stable trap using Laguerre-Gaussian. With Gaussian beams, the trapping efficiencies are low too. Upon increasing the size of the particle, the trapping efficiencies start to increase as seen in Table-4, Fig. 11 and Fig.12. $\mathrm{Q}_{\mathrm{zmax}}$ have higher values when Laguerre-Gaussian beams are used. Fig.8 shows that $\mathrm{Q}_{\mathrm{rmax}}$ values for Gaussian beam is always much higher than the Laguerre-Gaussian beam. When particle size is increased beyond $r=4 \mu \mathrm{m}$, the computations of trapping efficiencies become too time consuming.

Table-4

\begin{tabular}{|c|c|c|c|c|}
\hline \multirow{2}{*}{$\begin{array}{c}\text { Radius of the } \\
\text { particle, }(\mathbf{r})\end{array}$} & \multicolumn{2}{|c|}{ Gaussian beam } & \multicolumn{2}{c|}{$\begin{array}{c}\text { Laguerre-Gaussian } \\
\text { beam }\end{array}$} \\
\cline { 2 - 5 } & $\mathbf{Q}_{\text {zmax }}$ & $\mathbf{Q}_{\operatorname{rmax}}$ & $\mathbf{Q}_{\text {zmax }}$ & $\mathbf{Q}_{\operatorname{rmax}}$ \\
\hline 0.1 & 0.002163 & 0.003729 & N/A & N/A \\
\hline 0.2 & 0.01916 & 0.02213 & N/A & N/A \\
\hline 0.4 & 0.1036 & 0.1136 & N/A & N/A \\
\hline 0.5 & 0.1418 & 0.1745 & N/A & N/A \\
\hline 0.8 & 0.1905 & 0.1795 & N/A & N/A \\
\hline 1 & 0.2138 & 0.3945 & N/A & N/A \\
\hline 1.5 & 0.2506 & 0.4344 & 0.2574 & 0.1316 \\
\hline 2 & 0.2663 & 0.4759 & 0.3141 & 0.2418 \\
\hline 2.5 & 0.2662 & 0.4909 & 0.3384 & 0.2685 \\
\hline 3 & 0.2784 & 0.4614 & 0.3721 & 0.3069 \\
\hline 3.5 & 0.2531 & 0.3881 & 0.3504 & 0.2788 \\
\hline 4 & 0.2697 & 0.4378 & 0.3892 & 0.3147 \\
\hline 4.5 & 0.3294 & 0.2731 & 0.3294 & 0.2731 \\
\hline 5 & 0.2517 & 0.3047 & 0.3762 & 0.3004 \\
\hline 5.5 & 0.3122 & 0.2703 & 0.3122 & 0.2703 \\
\hline
\end{tabular}

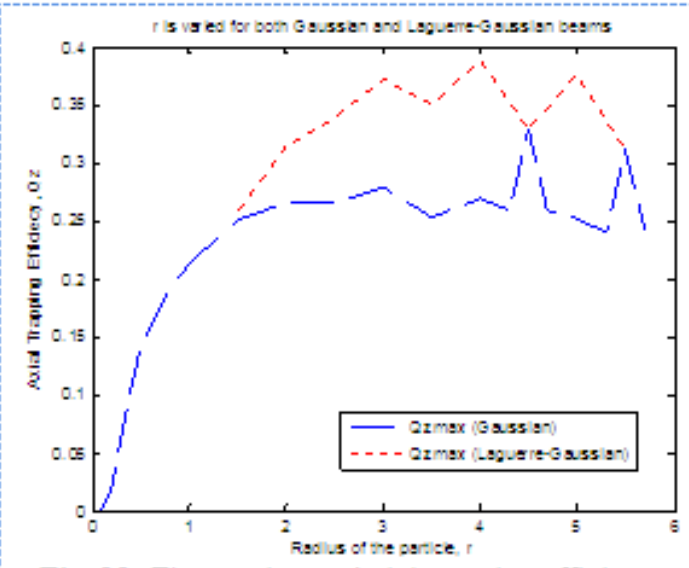

Fig. 11: The maximum Axial trapping efficiency $\left(Q_{\text {rmax }}\right)$ when the size of the particle ( $\left.r\right)$ is varied for both Gaussian beam and Laguerre-Gaussian beams.

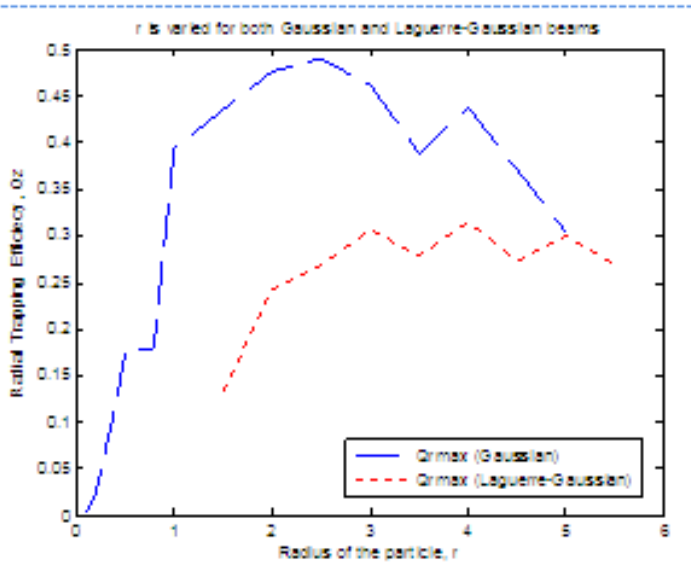

Fig. 12: The maximum Radial trapping efficiency $\left(Q_{\text {max }}\right)$ when the size of the particle $(r)$ is varied for both Gaussian beam and Laguerre-Gaussian beams.

\section{Conclusion}

In this paper, it has been numerically analyzed how trapping efficiencies of optical tweezers depend on the refractive index of the medium $\left(\mathrm{n}_{\mathrm{p}}\right)$, refractive index of the particle $\left(\mathrm{n}_{\mathrm{m}}\right)$, numerical aperture (NA) of the microscope objective and the size of the particle ( $\mathrm{r}$ ) being trapped. Simulations were performed for a variety of combinations of these four parameters to study how the trapping efficiencies are affected.

Investigation of trapping efficiency in Section-3 shows that Laguerre-Gaussian beam achieves much better axial trapping for the same conditions (beam power, particle size, refractive of the medium, refractive index of the particle, numerical aperture of the microscope objective) when compared to Gaussian beam. On the other hand, it can be also be observed that the radial trapping efficiency of the Laguerre-Gaussian beam is calculated to be always slightly less that that of an equivalent Gaussian beam. However, Gaussian beams are still used in optical tweezers to trap transparent particles where absorption effects are much smaller. Gaussian beam is also easier to produce and it gives higher intensity as losses in conversion to Laguerre-Gaussian 
(doughnut shaped) beam are avoided. The beam structure also allows the power of the laser beam to be focused into a small area. [1]

Although the goal of this paper was to analyze the behavior of trapping efficiencies of optical tweezers for two types of laser beams, there are still many additional factors left to be examined. For further research, trapping efficiencies of optical tweezers could be investigated for higher order laser beams like HermiteGaussian and Bessel beams. Also the trapping efficiencies could be analyzed for different shapes of particles being trapped.

\section{References}

[1]. Steffen Tallieu, Holographic optical trapping, master thesis, Ghent University, Ghent, Belgium, 2011-2012

[2]. Yuxiang Liu, Fiber optical tweezers for microscale and nanoscale particle manipulation and force sensing, doctoral dissertation, University of Maryland, College Park, Maryland, 2011

[3]. Aruna Ranaweera, Investigations with Optical Tweezers: Construction, Identification, and Control, doctoral dissertation, University of California, Santa Barbara, 2004

[4]. Valeria Garbin,Optical Tweezers for the study of microbubble dynamics in ultrasound, dissertation, The University of Trieste, Italy, 2005-6

[5]. Heckenberg, N. R., Friese, M. E. J., Nieminen, T. A., \& Rubinsztein-Dunlop, H. (2003), Mechanical effects of optical vortices, arXiv preprint physics/0312007

[6]. Timo A. Nieminen, Vincent L. Y. Loke, Alexander B. Stilgoe, Gregor Knőner, Agata M. Brańczyk, Norman R. Heckenberg and Halina Rubinsztein-Dunlop, Optical tweezers computational toolbox, Journal of Optics A 9, S196-S203 (2007)

[7]. S. Bayoudh, T. A. Nieminen, N. R. Heckenberg and H. Rubinsztein-Dunlop, Orientation of biological cells using plane-polarized Gaussian beam optical tweezers, Journal of Modern Optics 50(10), 1581.1590 (2003)

[8]. T. A. Nieminen, H. Rubinsztein-Dunlop and N. R. Heckenberg, Calculation of the T-matrix: general considerations and application of the point-matching method, Journal of Quantitative Spectroscopy and Radiative Transfer 79.80, 1019.1029 (2003)

[9]. T. A. Nieminen, H. Rubinsztein-Dunlop, N. R. Heckenberg and A. I. Bishop, Numerical modelling of optical trapping, Computer Physics Communications 142, 468-471 (2001) 